\title{
Olhar do enfermeiro na assistência de enfermagem do paciente autista e sua família
}

\author{
Nurses' perspective on the nursing care of autistic patients and their families \\ Perspectiva de las enfermeras sobre el cuidado de enfermería de los pacientes autistas y sus familias
}

Recebido: 03/09/2021 | Revisado: 13/09/2021 | Aceito: 29/10/2021 | Publicado: 01/11/2021

\author{
Andreza da Silva Fontinele \\ ORCID: https://orcid.org/0000-0002-3239-357X \\ Faculdade Estácio de Teresina, Brasil \\ E-mail: andrezacristynna@outlook.com \\ Angélica Gilderllany Sousa Silva \\ ORCID: https://orcid.org/0000-0003-3504-4161 \\ Faculdade Estácio de Teresina, Brasil \\ E-mail: angelicasousa967@gmail.com \\ Ingrid Gabrielle Ferreira Santos \\ ORCID: https://orcid.org/0000-0001-5347-0119 \\ Faculdade Estácio de Teresina, Brasil \\ E-mail: ingridgabrielle06@gmail.com \\ Joelma Muniz da Silva \\ ORCID: https://orcid.org/0000-0001-8242-7611 \\ Faculdade Estácio de Teresina, Brasil \\ E-mail: joelmamuniz19@hotmail.com \\ Juliete Machado Aguiar Bandeira \\ ORCID: https://orcid.org/0000-0002-2299-8014 \\ Faculdade Estácio de Teresina, Brasil \\ E-mail: julietebioanalise@gmail.com \\ Francilene Machado da Silva Gonçalves \\ ORCID: https://orcid.org/0000-0003-3157-5811 \\ Faculdade Estácio de Teresina, Brasil \\ E-mail: machadofrancilene37@gmail.com \\ Fabio Marcio de Sousa Castro \\ ORCID: https://orcid.org/0000-0002-7768-9892 \\ Faculdade Estácio de Teresina, Brasil \\ E-mail: fabiomscastro@gmail.com \\ Mayllane Marques Bezerra \\ ORCID: https://orcid.org/0000-0001-7213-8138 \\ Faculdade Estácio de Teresina, Brasil \\ E-mail: arraismayllane@gmail.com \\ Rosana Serejo dos Santos \\ ORCID: https://orcid.org/0000-0003-3084-7539 \\ Faculdade Estácio de Teresina, Brasil \\ E-mail: enf.rosanaserejo@gmail.com \\ Filipe Augusto de Freitas Soares \\ ORCID: https://orcid.org/0000-0003-0510-3968 \\ Faculdade Estácio de Teresina, Brasil \\ E-mail: filipe-freitas_2008@hotmail.com
}

\begin{abstract}
Resumo
Objetivo: Identificar na literatura os fatores relacionados ao papel do enfermeiro, dentro do contexto da assistência mental, em foque ao um cliente autista, com relação à importância do vínculo familiar. Metodologia: Trata-se de uma revisão integrativa da literatura, feita com base em artigos disponíveis na Biblioteca Virtual em Saúde - BVS. Resultados E Discussão: Dos 75 artigos encontrados, após os critérios de inclusão e exclusão, foram examinados e discutidos 10 artigos científicos nacionais, com a temática "Aspectos sociais e familiares da criança com Transtorno do Espectro Autista; Importância do diagnóstico precoce; Papel do Enfermeiro na Assistência ao cliente com Transtorno do Espectro Autista.". Conclusão: O autismo é uma temática desconhecida pela população leiga e esse desconhecimento vem prejudicando a inserção dessas crianças no contexto social e familiar. Isto compromete o desenvolvimento cognitivo e afetivo da criança. Assim sendo, a atuação do enfermeiro dentro da assistência ao cliente autista e sua família por meio da Sistematização da Assistência de Enfermagem (SAE) mostra-se de grande importância
\end{abstract}

Palavras-chave: Assistência de enfermagem; Família; Transtorno autístico.

\section{Abstract}

Objective: To identify, in the literature, factors related to the role of the nurse, within the context of mental care, focusing on an autistic client, in relation to the importance of the family bond. Methodology: This is an integrative literature 
review, based on articles available in the Virtual Health Library - VHL. Results and Discussion: Of the 75 articles found, after the inclusion and exclusion criteria, 10 national scientific articles were examined and discussed, with the theme "Social and family aspects of children with Autistic Spectrum Disorder; Importance of early diagnosis; Nurse's Role in Customer Care with Autistic Spectrum Disorder.". Conclusion: Autism is an unknown topic for the lay population and this lack of knowledge has been hindering the insertion of these children in the social and family context. This compromises the child's cognitive and affective development. Therefore, the role of nurses within the assistance to autistic clients and their families through the Systematization of Nursing Care (SAE) is of great importance.

Keywords: Nursing assistance; Family; Autistic disorder.

\section{Resumen}

Objetivo: Identificar, en la literatura, factores relacionados con el rol de la enfermera, en el contexto del cuidado mental, con foco en un cliente autista, en relación a la importancia del vínculo familiar. Metodología: Se trata de una revisión integradora de la literatura, basada en los artículos disponibles en la Biblioteca Virtual en Salud - BVS. Resultados y Discusión: De los 75 artículos encontrados, luego de los criterios de inclusión y exclusión, se examinaron y discutieron 10 artículos científicos nacionales, con el tema "Aspectos sociales y familiares de los niños con Trastorno del Espectro Autista; Importancia del diagnóstico precoz; Papel de la enfermera en la atención al cliente con trastorno del espectro autista". Conclusión: El autismo es un tema desconocido para la población laica y este desconocimiento ha venido dificultando la inserción de estos niños en el contexto social y familiar. Esto compromete el desarrollo cognitivo y afectivo del niño. Por tanto, el papel de las enfermeras dentro de la asistencia a los pacientes autistas y sus familias a través de la Sistematización de la Atención de Enfermería (SAE) es de gran importancia.

Palabras clave: Asistencia de enfermería; Familia; Trastorno autista.

\section{Introdução}

Resignando, outras doenças mentais ou comportamentais, o autismo é um assunto carregado de tabus e muito preconceito dentro da sociedade como também na saúde. O autismo passou a não ser mais retratado como "psicose infantil”, no período do ano 1980. Dito isso, o mesmo foi nomeado como um Transtorno Invasivo do Desenvolvimento (TID) no Manual de Saúde Mental III - (DSM-3). Na atualidade, a última atualização do Manual de Saúde Mental V - DSM-5, o autismo, a Síndrome de Asperger e o transtorno invasivo do desenvolvimento inespecífico passaram a serem considerados dentro de um diagnóstico único, os Transtornos do Espectro do Autismo- TEA (Brasil, 2015).

O TEA, conforme o Manual de Diagnóstico e Estatístico de Transtorno Mental (DSM-V) é um distúrbio neurológico caracterizado por comprometimento da interação social, diálogo verbal e não verbal, pelo comportamento restrito, repetitivo e no uso da imaginação (Araújo, Nascimento \& Dutra, 2019).

Segundo a Organização Pan-Americana da Saúde-OPAS (2019), o TEA era considerado raro, porém, no panorama mundial é possível observar um aumento da taxa de prevalência na última década, de aproximadamente de 4 a cada 10000 crianças para 1 a cada 68 crianças. No Brasil, a estimativa é que existam 2 milhões de pessoas autistas, e a taxa de prevalência passou de 4 casos a cada 1000 crianças para aproximadamente, 1 caso a cada 160 crianças. O transtorno acomete quatro vezes mais pessoas do sexo masculino, e não tem diferença entre raças. Entretanto, meninas quando diagnosticadas são mais prejudicadas.

No entanto, o autismo se dá em torno dos dois primeiros anos de vida e aqueles com quoeficiente de inteligência (QI) maior e capazes de articular, terá prognóstico mais positivo. Na vida adulta, os problemas de comunicação e socialização tendem a prosseguir, e somente uma pequena parcela consegue independência. Até o momento, não se alcançou a cura, sendo levado em consideração o modelo terapêutico, em referência tende ajudá-los a obter independência para atividades diárias, como utilizar uma peça de roupa e se higienizar (Santos, Santos \& Santos, 2019).

Neste aspecto, quando o cliente é diagnosticado com a patologia autismo, a relação de necessidade de um apoio multidisciplinar é imensa em papel no acompanhamento pessoal, não só focado no mesmo, mas abordando a família também. A enfermagem se faz de extrema importância na prestação desse cuidado, auxiliando na promoção, prevenção e no desenvolvimento da criança. Com parâmetros, no qual o profissional desenvolvera medidas alternativas incluídos familiares, facilitando o acesso às informações sobre o transtorno e procurando promover ações que proporcionem o bem-estar do paciente 
e do seu familiar ligando esses aos serviços de saúde essenciais (Ebert, Lorenzini \& Silva, 2015).

Diante das dificuldades enfrentadas, são apontados na cognição e na socialização, como o papel da equipe de enfermagem fundamental para auxiliar no tratamento, tendo conhecimento suficiente para perceber os primeiros sinais e sintomas desse distúrbio, devendo estar atento à valorização da verbalização, que sua ação é de inserção é importante no processo de cuidar, pois, a qualificação profissional do enfermeiro ainda não está preparada totalmente para acolher um cliente autista, o que impede um diagnóstico precoce, e aumenta o estresse familiar em intermédio da busca de um melhor tratamento (Silva, 2018).

Diante deste exposto, o presente estudo teve o objetivo de identificar na literatura os fatores relacionados ao papel do enfermeiro, dentro do contexto da assistência mental, em foque ao um cliente autista, com relação à importância do vínculo familiar. O olhar da equipe de enfermagem é de imensa valia nesta atuação, sendo possível o aprofundamento de práticas e a inclusão no modelo assistencial.

\section{Metodologia}

A busca foi realizada via Biblioteca Virtual de Saúde (BVS), conforme as bases de dados base de dados Literatura Latino-Americana e do Caribe em Ciências da Saúde (LILACS), Medical Literature Analysis and Retrievel System Online (Medline) e National Library of Medicine National Institutes of Health (PubMed).

Com isso, foi utilizado o primeiro segmento para construção da prática científica, que simplifica o passado da literatura empírica ou teórica, com o objetivo de prover uma abrangente cognição de um fenômeno inato. Esse método de pesquisa tem finalidade de construir uma investigação em pauta no conhecimento já arquitetado em pesquisas em relação a um assunto determinado. E proporciona estes resultados de forma sistematizada com a finalidade de obter um conhecimento para execução das práticas profissionais embasadas em evidências clínicas (Botelho, Cunha \& Macedo, 2011).

Assim, para a escolha dos artigos, realizou-se os seguintes Descritores em Saúde (DeCS): "Transtorno Autístico", “Assistência Enfermagem" e "Família", e o operador booleano "AND", onde obteve um total de 75 artigos. Posto isto, os perfis de inclusão delineadas para a seleção dos artigos foram: artigos publicados em português; artigos na íntegra que retrata a temática referente à atuação do enfermeiro na assistência do paciente autista, e a importância do apoio familiar e os artigos publicados e indexados nos referidos bancos de dados nos últimos cincos anos. Já os critérios de exclusão foram: artigos com duplicidade durante a busca, teses, dissertações, artigos de opinião, de relatos de casos, de revisão, artigos que não abordavam o assunto, aqueles que não eram disponibilizados gratuitamente.

Em seguida, obtiveram 20 estudos. Sendo analisados criteriosamente de acordo com os critérios já citados. Os 8 artigos excluídos, abordavam outras temáticas, 2 não estavam disponíveis para acesso, 3 sendo revisão sistemática, desse modo, selecionados 10 trabalhos. Para isso, utilizou-se um roteiro de coleta de dados, objetivando extrair informações de identificação de período, focando nos objetivos, aspectos metodológicos, principais resultados e conclusões. Depois desta etapa, procedeu-se uma análise profunda dos artigos, utilizando de metodologia unicamente qualitativa, visando à identificação e interpretação das ideias centrais dos artigos e posteriormente, agrupar tais ideias em núcleos ou categorias de significados.

\section{Resultados e Discussão}

Conforme retratado anteriormente, a amostra desta revisão foi composta por 07 artigos científicos nacionais. Na tabela abaixo, estão relacionados às publicações analisadas para compor esta revisão de literatura. 
Tabela 1: Descrição dos Artigos selecionados segundo: Autor, Ano, Periódico, Desenho Metodológico, Objetivo e Conclusão.

\begin{tabular}{|c|c|c|c|c|c|}
\hline Autor (a) & Ano & Periódico & $\begin{array}{c}\text { Desenho } \\
\text { Metodológico }\end{array}$ & Objetivo & Conclusão \\
\hline $\begin{array}{l}\text { Menotti, } \\
\text { Domeniconi } \\
\text { \& Benitez }\end{array}$ & 2019 & $\begin{array}{c}\text { Psicologia } \\
\text { Escolar e } \\
\text { Educaciona } \\
1\end{array}$ & $\begin{array}{l}\text { Linha de base } \\
\text { múltipla }\end{array}$ & $\begin{array}{l}\text { Avaliar a eficácia de um pacote } \\
\text { instrucional para o ensino de } \\
\text { leitura de quinze palavras } \\
\text { dissílabas (isoladas) para crianças } \\
\text { com TEA, baseado no modelo de } \\
\text { leitura como rede de relações. }\end{array}$ & $\begin{array}{l}\text { As crianças obtiveram } \\
\text { ganhos na leitura das } \\
\text { palavras ensinadas e os pais } \\
\text { aprenderam a utilizar } \\
\text { reforço e dica durante os } \\
\text { jogos. } \\
\end{array}$ \\
\hline $\begin{array}{l}\text { Hofzmann, } \\
\text { Perondi e } \\
\text { Menegaz }\end{array}$ & 2019 & $\begin{array}{c}\text { Enferm. } \\
\text { Foco }\end{array}$ & $\begin{array}{c}\text { Pesquisa } \\
\text { qualitativa, } \\
\text { através de Grupo } \\
\text { Focal } \\
\end{array}$ & $\begin{array}{c}\text { Conhecer a experiência dos } \\
\text { familiares no convívio de crianças } \\
\text { com TEA }\end{array}$ & $\begin{array}{l}\text { Necessidade do apoio dos } \\
\text { profissionais de saúde no } \\
\text { suporte dos cuidados } \\
\text { prestado a estas crianças. }\end{array}$ \\
\hline $\begin{array}{l}\text { Santos, Santos } \\
\text { e Londero }\end{array}$ & 2019 & $\begin{array}{l}\text { Distúrb } \\
\text { Comum }\end{array}$ & caso clínico & $\begin{array}{c}\text { Analisar o processo de } \\
\text { reidealização do filho com } \\
\text { autismo na clínica singular da } \\
\text { criança e sua família }\end{array}$ & $\begin{array}{l}\text { A partir da escuta da mãe e } \\
\text { da descoberta das } \\
\text { potencialidades linguística, } \\
\text { cognitiva e subjetiva }\end{array}$ \\
\hline $\begin{array}{l}\text { Faro, Santos e } \\
\text { Bosa. }\end{array}$ & 2019 & Psico & $\begin{array}{l}\text { Quantitativa e } \\
\text { descritiva, }\end{array}$ & $\begin{array}{l}\text { Foi comparar dois grupos de mães } \\
\text { de crianças com autismo (com e } \\
\text { sem estresse). }\end{array}$ & $\begin{array}{c}\text { Mães com estresse tiveram } \\
\text { quase o dobro de percepção } \\
\text { de sobrecarga, enquanto as } \\
\text { sem estresse perceberam } \\
\text { maior suporte familiar. }\end{array}$ \\
\hline $\begin{array}{l}\text { Mapelli, } \\
\text { Barbieri e } \\
\text { Castro }\end{array}$ & 2018 & $\begin{array}{l}\text { Esc. Anna } \\
\text { Nery }\end{array}$ & $\begin{array}{l}\text { Pesquisa } \\
\text { descritiva, } \\
\text { qualitativa }\end{array}$ & $\begin{array}{c}\text { Conhecer a experiência da família } \\
\text { no cuidado da criança com } \\
\text { Transtorno do Espectro Autista }\end{array}$ & $\begin{array}{l}\text { O cuidado da criança com } \\
\text { transtorno do espectro } \\
\text { autista potencializa } \\
\text { estratégias de } \\
\text { fortalecimento e adaptação. }\end{array}$ \\
\hline Nobre e Souza & 2018 & $\begin{array}{l}\text { Rev baiana } \\
\text { enferm }\end{array}$ & $\begin{array}{l}\text { Estudo } \\
\text { descritivo, } \\
\text { qualitativo }\end{array}$ & $\begin{array}{c}\text { Desvelar as vivências trazidas por } \\
\text { pais e/ou cuidadores de crianças } \\
\text { com autismo em um serviço de } \\
\text { Plantão Psicológico. }\end{array}$ & $\begin{array}{l}\text { As dificuldades com os } \\
\text { cuidados com as crianças e } \\
\text { o isolamento social foram } \\
\text { eixos comuns que } \\
\text { compartilharam. }\end{array}$ \\
\hline $\begin{array}{l}\text { Constantinidis } \\
\text {, Silva e } \\
\text { Ribeiro }\end{array}$ & 2018 & Psico-USF & $\begin{array}{l}\text { Pesquisa } \\
\text { qualitativa }\end{array}$ & $\begin{array}{l}\text { Compreender a vivência de mães } \\
\text { de crianças com autismo, com } \\
\text { base em entrevistas e estudos } \\
\text { existentes, situando o debate } \\
\text { teórico em torno desse processo. }\end{array}$ & $\begin{array}{c}\text { Importância do diagnóstico } \\
\text { como norteador das ações } \\
\text { dessas mães e a resistência } \\
\text { dos profissionais em } \\
\text { fornecê-lo } \\
\end{array}$ \\
\hline Seize e Borza, & 2017 & Psico-USF & $\begin{array}{l}\text { Revisão de } \\
\text { artigos }\end{array}$ & $\begin{array}{l}\text { Identificar os instrumentos } \\
\text { disponíveis para rastreamento dos } \\
\text { sinais do autismo em crianças } \\
\text { com até } 36 \text { meses de idade. }\end{array}$ & $\begin{array}{l}\text { Relevância da identificação } \\
\text { e do diagnóstico precoce do } \\
\text { autismo para a melhoria da } \\
\text { qualidade de vida do sujeito }\end{array}$ \\
\hline $\begin{array}{l}\text { Corrêa e } \\
\text { Queiroz }\end{array}$ & 2017 & $\begin{array}{c}\text { Ciências \& } \\
\text { Cognição }\end{array}$ & $\begin{array}{l}\text { A pesquisa é de } \\
\text { cunho } \\
\text { descritivo. }\end{array}$ & $\begin{array}{l}\text { Analisar as trocas sociais, } \\
\text { formadas por crianças com } \\
\text { diagnóstico de TEA, submetidas a } \\
\text { um programa de intervenção } \\
\text { precoce, e suas mães. }\end{array}$ & $\begin{array}{c}\text { Conclui-se que com } \\
\text { sistema de acolhimento e } \\
\text { orientação familiar é } \\
\text { possível criar ambientes de } \\
\text { desenvolvimento } \\
\text { adequados. }\end{array}$ \\
\hline $\begin{array}{l}\text { Silva, Lima, e } \\
\text { Carvalho }\end{array}$ & 2017 & $\begin{array}{l}\text { Rev enferm } \\
\text { UFPE }\end{array}$ & $\begin{array}{c}\text { Estudo } \\
\text { avaliativo, } \\
\text { descritivo e } \\
\text { exploratório, } \\
\text { com abordagem } \\
\text { quantitativa e } \\
\text { qualitativa. }\end{array}$ & $\begin{array}{c}\text { Avaliar a qualidade de vida de } \\
\text { crianças que tão no espectro do } \\
\text { autismo. }\end{array}$ & $\begin{array}{c}\text { Os pais ou cuidadores } \\
\text { dessas crianças acreditam } \\
\text { que os mesmos possam ter } \\
\text { boa qualidade de vida e } \\
\text { desenvolver } \\
\text { comportamentos comuns a } \\
\text { todas as crianças. }\end{array}$ \\
\hline
\end{tabular}

Fonte: Autores (2020).

Após minuciosa leitura de cada artigo e de sua análise de conteúdo, foi possível a identificação de três núcleos de significado: Aspectos sociais e familiares da criança Autista; Importância do diagnóstico precoce; Papel do Enfermeiro na Assistência ao cliente com Transtorno do Espectro Autista. Cabe salientar que embora sejam núcleos que enfoquem abordagens distintas, os temas se complementam e facilitam a análise da temática pesquisada. 


\section{Aspectos sociais e familiares da criança Autista.}

Segundo o DSM-5, o transtorno do espectro autista (TEA), quando diagnosticado em uma criança, pode ocasionar em sua família, sentimentos que partem da aceitação ou desafio prazeroso até sentimentos como: preocupação, sofrimento, negação, sensação de impotência e previsão de momentos turbulentos. Entretanto, o tipo de sentimento expressado também irá depender do grau de severidade do autismo (leve, moderado ou severo) ou ao nível de aprendizado dos familiares relacionado ao autismo (Hofzmann et al.,2019).

Desse modo, o desconhecimento ou ainda o conceito equivocado a respeito do autismo, evidencia a relevância da propagação de informações em relação ao TEA para a sociedade, sobretudo para população leiga, que pode vir a conviver diariamente e diretamente com um autista. Para que a criança autista seja acolhida, faz-se necessário que o conceito, as características e as formas de tratamento sejam conhecidas por todos, facilitando o acolhimento pela família, especialistas e sociedade, gerando com que esse indivíduo receba o apoio necessário para seu desenvolvimento cognitivo, pessoal e social (Corrêa \& Queiroz, 2017).

Os comprometimentos advindos do autismo trazem impactos aos familiares e ao autista, que necessitam se ajustar às novas demandas e às exigências advindas da condição de deficiência da criança. Apesar da pouca cognição nessa temática, fica clara a necessidade de realização de mais estudos a respeito da atenção voltada para essas crianças e todos ao seu redor, a fim de que os profissionais da saúde que estejam envolvidos na assistência, consigam de forma baseada no conhecimento científico, prestar uma melhor assistência às famílias e aos portadores do TEA, elevando os sentimentos de altivez no enfrentamento imposto pelas dificuldades relacionadas ao transtorno (Faro et al., 2019).

\section{Importância do diagnóstico precoce.}

A análise da pesquisa de Mapelli et al., (2018) identifica que o TEA é descrito como um distúrbio relacionado ao neurodesenvolvimento e geralmente tem sua manifestação na primeira infância. Sendo subdivido em dois domínios: um associado a dificuldades de comunicação e entrosamento com outras pessoas. Já o outro está relacionado aos comportamentos restritivos e repetitivos. No entanto, o Governo Brasileiro apresenta a busca de meios alternativos baseados nos direitos sustentados pelo instituto Nacional de Proteção dos Direitos das Pessoas com Transtorno do Espectro Autista, com finalidade de garantir o diagnóstico precoce, atendimento multiprofissional e acesso a informações que auxiliam no prognostico e tratamento.

Dessa forma, observar sinais e sintomas do autismo precocemente, pode-se chegar a um diagnóstico igualmente prévio, isso influi positivamente para uma melhora no bem-estar e no progresso de vida dessa criança. Importantes estudos nas áreas da Psicologia, Psiquiatria e Neuropsiquiatria já foram publicados em outros países como os Estados Unidos, Canadá, Austrália entre outros, mostrando como é feito esse diagnóstico precoce (Santos et al.,2019).

Diante disso, foi originado a escala de M-CHAT que tem como objetivo realizar o rastreamento para detectar indícios desse transtorno com pessoas de 18 a 24 meses. Essa técnica tem um leque no ponto norteador, por não ter a necessidade de equipamentos e laboratório, para o diagnóstico. O método se baseia em cima de um questionário, o mesmo é aplicado nos cuidadores ou nos pais das crianças, contendo uma alternativa eficiente e sem custos financeiros para obter o diagnóstico do TEA precocemente (Menotti et al., 2019).

Um estudo realizado por Seize e Borza (2017), na Europa, pode-se identificar 11 instrumentos para rastrear manifestações de sintomas e sinais do autismo em crianças com até 3 anos de idade e foram divididos em 2 níveis. Os instrumentos do nível 1, utilizados para a população em geral, com a função de detectar crianças com sinais de risco de autismo, usaram o método em formato de questionário, com tamanho variando entre 9 e 63 itens, indicando esse formato o mais adequado para uso na população, onde os que prestavam cuidados em casa dessas crianças eram os respondentes.

Enquanto no nível 2, era para ser utilizado para a população de risco, ou seja, crianças que já apresentem visivelmente 
sinais claros de problemas ao decorrer do seu crescimento. Com isso, foram utilizado instrumentos para observar os pais, o POEMS, é um dos instrumento que tem função de analisar perspectivas de cunho social e de comunicação, assim como, domínio do movimento motor grosso, é um deles e também um dos mais utilizados, geralmente por profissionais que possui um maior entendimento sobre esse assunto, o BISCUIT-I, é um método que utiliza-se uma escala onde aplica-se aos trabalhadores de saúde que são dessa área, ao final apresentou consistência adequada em seus três fatores no estudo realizado por (Seize \& Borza, 2017).

Diante do exposto, foi possível identificar que a insuficiência de instrumentos que auxilia na identificação dos sinais prévios do autismo, resulta em diagnósticos tardios e investigações imprecisas nas condições de saúde dessas crianças. Dificultando assim, o crescimento e desenvolvimento dos mesmos (Hofzmann et al.,2019). No Brasil, existe também uma escassez em estudos nessa temática, o que propõe uma reflexão por parte dos profissionais da área de saúde mental brasileira (Santos et al.,2019).

\section{Papel do Enfermeiro na Assistência ao cliente com Transtorno do Espectro Autista.}

$\mathrm{O}$ apoio do enfermeiro na assistência ao autista é um suporte indispensável e de suma importância tanta para essas crianças, como para as mães/cuidadores. Os mesmos necessitam desse apoio em momentos distintos, pois o autismo requer mudanças e adaptações na vida desses pais. O enfermeiro, como membro da equipe multiprofissional, é um dos principais responsável por acompanhar e avaliar o desenvolvimento infantil, a fim de detectar precocemente qualquer anormalidade e tomar as medidas resolutivas para a melhoramento da bem-estar da pessoa portadora de TEA e de todos ao seu redor (Constantinidis et al., 2018).

Em vista disto, a equipe de enfermagem deve atentar-se às singularidades dos indivíduos e suas respectivas necessidades, prestando uma assistência íntegra e de qualidade que atenda a todas as demandas de cuidado dos autistas e de seus familiares, contribuindo para o fortalecimento e ampliação dos seus laços relacionais. O enfermeiro envolto, pela sua competência em cuidar não só do doente, é um profissional capaz de inserir-se no cuidado em domicílio e contribuir com a família na organização da dinâmica e cuidada familiar (Silva et al., 2017).

Neste contexto, para o enfermeiro realizar uma assistência de qualificada faz se necessário à implementação da SAE, com o uso de uma teoria de enfermagem, buscando identificar problemas reais e potenciais junto ao cliente autista, abordando nas consultas de enfermagem e na assistência prestada a importância de apaga-se a pequenos gestos e sintomas que podem ser um risco para o autismo. Tendo com intuito de criar um plano assistencial centrado nas necessidades do paciente, envolvendo o mesmo neste planejamento (Faro et al., 2019).

Portanto, o impacto do autismo, podendo ser amenizado quando se entreposto por uma base de apoio familiar e social, com estratégias efetivas de enfrentamento e pela qualidade do sistema de saúde que acolhe os pais ou responsáveis. Com isso, é indispensável que os profissionais, cruzarem pelo processo de reciclagem do conhecimento as crianças autistas, no que diz respeito ao diagnóstico, tratamento e acompanhamento, bem como a identificação das suas necessidades para se aplicar uma intervenção que contribua para a melhoria do crescimento e desenvolvimento das crianças autistas, o que certamente produzirá maior segurança as famílias (Constantinidis et al., 2018).

Neste aspecto, o papel do enfermeiro na assistência ao cliente portador de transtorno do espectro autista é formar um elo entre o paciente e aos seus cuidadores. Ao se depararem com os pacientes autistas, possa-se fazer presente previamente, as intervenções adequadas e as orientações cabíveis aos familiares/cuidadores. Com isso, possa-se aumentar as chances de as crianças obterem desenvolvimento cognitivo, pessoal e social. E diante de muitos preconceitos e as poucas abordagens científicas sobre o tema, o enfermeiro deve buscar diante dos pacientes autistas e dos seus familiares, cuidar, acalentar e produzir novas formas de saber e fazer em enfermagem (Mapelli et al., 2018).

Neves et al., (2020) aponta em seus estudos a necessidade da reflexão de que o autista não é um indivíduo portador de 
uma doença que limita a capacidade de interagir socialmente, mas, sim, alguém que vive em seu eu particular. Diante disso, o acompanhamento e cuidado à pessoa com TEA exigem, dentre outras coisas uma reestruturação familiar, através de ajuda profissional. Corroborando com Neves et., (2020) o estudo de Silva et al., (2020) conclui que assistir a criança com distúrbio mental constitui se em um grande desafio, já que nesta área não temos enfermeiros especializados. Com estes instrumentos a nossa visão do problema se ampliou, foi possível identificar a necessidade de ensinar passo a passo uma nova habilidade para a criança, facilitando assim a sua compreensão.

De acordo com Araújo (2020), O papel do enfermeiro diz respeito à detecção dos primeiros sinais e sintomas e referenciamento da criança e a família para que a equipe médica realize o diagnóstico completo. Deste modo, destaca-se a importância do enfermeiro buscar conhecimento acerca do TEA, uma vez que a literatura aponta fragilidade e dificuldades referentes à detecção precoce da patologia, implementação de uma assistência singular e de qualidade, tanto para a criança quanto para a família.

\section{Conclusão}

Tendo em vista, o autismo é uma temática desconhecida pela população leiga e esse desconhecimento vem prejudicando a inserção dessas crianças no contexto social e familiar. Isto compromete o desenvolvimento cognitivo e afetivo da criança. Sendo visto a suma importância da atuação do enfermeiro dentro da assistência ao cliente autista e sua família, com a introdução da SAE. No entanto, é possível observar uma restrição subsequente de alguns profissionais nesta assistência e uma carência no elo familiar.

O enfermeiro na assistência ao cliente com TEA tem o papel de formar um elo entre a criança e aos pais/cuidadores, buscar realizar intervenções adequadas e as orientações cabíveis aos familiares/cuidadores. Atentar-se às singularidades dos indivíduos e suas respectivas necessidades, prestando uma assistência íntegra e de qualidade que atenda a todas as demandas de cuidado dos autistas e suas famílias. As ações de enfermagem devem visar o desenvolvimento cognitivo, pessoal e social do autista.

\section{Referências}

Araújo, C. M., Nascimento, J. S., \& Dutra, W. L. (2019). O papel do enfermeiro na assistência à criança autista. Revista Brasileira Interdisciplinar de Saúde. $1(3)$.

Araújo, M. G. O papel do enfermeiro no apoio à criança autista. 2020. Monografia (Graduação em Enfermagem) - Faculdade de Ciências da Educação e Saúde, Centro Universitário da Brasília, Brasília, 2020.

Botelho, L. L. R., Cunha, C. D. A., \& Macedo, M. (2011). The integrative review method in organizational studies. Rev Eletr Gestão Soc, 5(11), 121-36.

Brasil. Ministério da Saúde. (2015) Linha de cuidado para a atenção às pessoas com transtornos do espectro do autismo e suas famílias na rede de atenção psicossocial do sistema único de saúde. https://bvsms.saude.gov.br/bvs/publicacoes/linha_cuidado_atencao_pessoas_transtorno.pdf.

Constantinidis, T. C., Silva, L. C. D., \& Ribeiro, M. C. C. (2018). "Todo mundo quer ter um filho perfeito": vivências de mães de crianças com autismo. PsicoUSF, 23, 47-58.

Corrêa, M. C. C. B., \& Queiroz, S. S. D. (2017). A família é o melhor recurso da criança: análise das trocas sociais entre mães e crianças com transtorno do espectro do autismo. Ciênc. cogn, 41-62.

Ebert, M., Lorenzini, E., \& Silva, E. F. D. (2015). Mães de crianças com transtorno autístico: percepções e trajetóriasa. Revista Gaúcha de Enfermagem, 36, 4955 .

Faro, K. C. A., Santos, R. B., Bosa, C. A., Wagner, A., \& da Costa Silva, S. S. (2019). Autismo e mães com e sem estresse: análise da sobrecarga materna e do suporte familiar. Psico, 50(2), e30080-e30080.

Hofzmann, R. R, Perondi, M., Menegaz, J., Lopes, S. G. R., \& Borges, D. S (2019). Experiência dos familiares no convívio de crianças com transtorno do espectro autista (TEA). Enfermagem em Foco, 10(2).

Mapelli, L. D., Barbieri, M. C., Castro, G. V. D. Z. B., Bonelli, M. A., Wernet, M., \& Dupas, G. (2018). Criança com transtorno do espectro autista: o cuidado da família. Escola Anna Nery, 22. 
Research, Society and Development, v. 10, n. 14, e246101420229, 2021

(CC BY 4.0) | ISSN 2525-3409 | DOI: http://dx.doi.org/10.33448/rsd-v10i14.20229

Menotti, A. R. S., Domeniconi, C., \& Benitez, P. (2019). Activities implemented by parents to teach reading their children with autism. Psicologia Escolar e Educacional, 23.

Neves, K. do C., Felix, D. P. da S.., Ribeiro, W. A., Fassarella, B. P. A., \& Silva, A. A da. (2020). Acolhendo a pessoa com transtorno do espectro autista: um desafio para o cuidado de enfermagem. Research, Society and Development, 9 (8), e941986742. https://doi.org/10.33448/rsd-v9i8.6742

Nobre, D. S., \& Souza, A. M. (2018). Vivências de pais e/ou cuidadores de crianças com autismo em um serviço de plantão psicológico. Revista Baiana de Enfermagem, 32 .

OPAS. (2019). Folha informativa (2019) - Transtorno do espectro autista. Organização Pan-Americana Da Saúde (OPAS). https://www.paho.org/bra/index.php?Itemid=1098.

Santos, N. K., Santos, J. A. M., Santos, C. da P., \& Lima, V. P. (2019). Assistência de enfermagem ao paciente autista. Revista De Saúde Dom Alberto, 3(1), 17-29.

Santos, T. D., dos Santos, D. R., Londero, A. D., \& de Souza, A. P. R. (2019). Luto e Reidealização na Clínica da Infância: Estudo de um Caso. Distúrbios da Comunicação, 31(2), 207-216.

Seize, M. D. M., \& Borsa, J. C. (2017). Instrumentos para rastreamento de sinais precoces do autismo: revisão sistemática. Psico-USF, 22 , 161-176.

Silva, M. de L.., Silva, M. P. B., Cunha, R. de C. da S.., Leite, AC, Lima, V. F. D. S, Sousa, F. C. dos S. S., Santos, J. R. F. de M., Fernandes, J. M., Mello, E. C. de A., Coelho, R. K. S, Morais, L. S. F., Moura, L. C. de., Oliveira. J. S. de, \& Rosenstock, K. I. V. (2020). Assistência de enfermagem frente à seletividade alimentar de crianças autistas: revisão da literatura. Pesquisa, Sociedade e Desenvolvimento, 9(12), e37091211020. https://doi.org/10.33448/rsd-v9i12.11020.

Silva, R. G. O. (2018). Transtorno do espectro autismo-tea: assistência de enfermagem ao paciente com suspeita ou diagnóstico de autismo. Trabalho de Graduação em Enfermagem, p. 5, apresentado em 12 Dez. 2018.

Silva, R. N. A., Lima, A. F. A, Carvalho, F. S. S. C., da Silva, F. L., de Melo Vilanova, J., \& dos Santos, E. P. (2017). Avaliação da qualidade de vida de crianças que estão no espectro do autismo. Revista de Enfermagem UFPE on line, 11(9), 3461-3470. 